\title{
El urólogo como generalista de la gente mayor
}

\author{
F.J. Solé Balcells \\ Servicio de Urología. Fundación Puigvert. Barcelona.
}

Actas Urol Esp 2006; 30 (2): 103-109

$\mathrm{E}$ s evidente que actualmente en los países en buen desarrollo económico cada vez se viven más años, se alarga la duración de la vida. La primera revolución en este sentido se produjo a principios del siglo XX, gracias a la disminución de la mortalidad infantil, al lograr curar muchas de las enfermedades infecciosas gracias a los antibióticos y evitarlas mediante las vacunaciones. En aquel período el objetivo podía ser definido como "lograr que un mayor número de personas llegaran a la vejez". En este momento vivimos una segunda revolución, con el objetivo de retrasar el envejecimiento, es decir "lograr que un mayor número de viejos sean más viejos y al mismo tiempo más jóvenes en relación a su calidad de vida".

La expectativa de vida, es decir el número de años que un individuo al nacer puede esperar llegar a vivir, es actualmente en España de 75,3 años para los varones y de 82,5 años para las mujeres, mientras que hace sólo 100 años la expectativa de vida era sólo de 42 años. Las previsiones, según los estadísticos, señalan que los nacidos en la actualidad tendrán como expectativa de vida unos 100 años.

Pero, ¿qué es envejecer?. En forma simple podríamos definirlo como "hacerse viejo". En inglés se distingue entre "aging" y "senescence". Aging es sinónimo de envejecimiento acumulando años con buena salud y senescence es envejecer con degradación, lo que en nuestro idioma podríamos definir como senectud. En forma simplista también podríamos clasificar tres modos de envejecer: a) envejecer con éxito, o sea sin pérdidas, b) envejecer "normalmente", con lógicas pérdidas por la edad y c) envejecer patológico, con enfermedad concomitante. En realidad, envejecer no es en sí mismo una enfermedad, es un proceso fisiológico, pero que en la mayor parte de los casos convierte a los adultos sanos en seres frágiles.
La actuación contra el envejecimiento o "medicina antiaging" es un nuevo enfoque de la medicina preventiva y es muy diferente del contenido de la geriatría. La geriatría es responsable del tratamiento de las patologías y deficiencias físicas o psíquicas de los mayores de 65 años. El objetivo de la medicina anti-envejecimiento -una nueva especialidad- estriba en retrasar la edad biológica de las arterias, de los huesos, de los músculos, de las glándulas endocrinas, del sistema nervioso y del sistema inmunitario, a partir los 40 a 45 años.

Los urólogos debemos ser especialistas en medicina anti-envejecimiento, dado que cada vez vienen a nuestras consultas varones más jóvenes gracias a los chequeos por el PSA, y por otra parte, por el papel que en este momento se da a la prevención de la carcinogénesis, por lo que, sin duda, los consejos de cómo retrasar el envejecimiento pueden contribuir a vivir una vejez más saludable.

El urólogo, al tener su primer contacto con el varón que consulta para chequeo de su próstata, debe investigar -como generalista- la posible hipertensión arterial, la diabetes tipo II, la hiperlipidemia y asimismo preguntar sobre su función eréctil y orientarle de cómo debe luchar contra los radicales libres, con una buena dieta mediterránea y el empleo de antioxidantes, así como el posible tratamiento de su disfunción eréctil o del hipogonadismo.

$\mathrm{El}$ envejecimiento empieza antes de nacer. Sin lugar a dudas existe un factor genético, ligado al genoma. Es la "herencia de la longevidad". Hay familias en las que los nonagenarios son más abundantes que en otras familias de la misma raza y sociedad. Pero además se sabe actualmente, en relación a la esperanza de vida, que hay que tener en cuenta factores del "entorno", que 
serán transmitidos a las siguientes generaciones. Es la llamada "herencia epigenética". Un ejemplo que ha renovado el interés hacia la teoría de Lamarque (1744-1829) -sobre la herencia de las características adquiridas- ha sido el registro de los nacimientos habidos durante la gran escasez de alimentos en Holanda en los años 1944-1945, en plena guerra mundial, en que nacieron niños con escaso peso. Se ha visto que al llegar a la edad adulta estos niños presentan una mayor prevalencia de diabetes, obesidad, enfermedades coronarias, cáncer de mama, etc. y lo más sorprendente es que sus hijos, o sea los nietos de la generación del hambre, presentan los mismos problemas. La herencia epigenética explica que el entorno influye en el genoma, en apoyo de la teoría evolucionista de Darwin.

Los nacidos por alteraciones en la nutrición durante la vida intrauterina presentan un desarrollo precoz de arterioesclerosis debido a que el bajo peso al nacer da lugar a un trastorno de la función endotelial, como paso previo a la arterioesclerosis, que puede presentarse ya a los 9-11 años de edad. Eso no ocurre en el "prematuro", o sea en el nacido con bajo peso fetal, por parto anticipado.

Y, ¿cómo retrasar el envejecimiento?. Cuatros teorías son los que actualmente se citan como las principales a tener en cuenta, teóricas algunas y otras con fines prácticos:

1. Manipulaciones genéticas.

2. Investigación sobre Telómeros y Telomerasa.

3. Importancia de la restricción calórica alimenticia.

4. Prevención del daño oxidativo.

En esta exposición nos vamos a limitar a considerar el daño oxidativo y la importancia de la restricción calórica alimenticia.

La teoría de los radicales libres (o teoría de Harman, 1954) considera que a nivel celular los micronutrientes son responsables de la producción de energía - precisa para todos los procesos biológicos- y de la formación de radicales libres, moléculas con electrones no aparejados en su órbita externa, y capaces de degradar los glúcidos, los lípidos, las proteínas, el mismo DNA, así como las células, los tejidos y los órganos.
La vida de Denhan Harman es sumamente interesante. Inició sus estudios sobre radiación química en el laboratorio de la compañía SHELL en 1940, completando su formación en medicina interna y en química en la Universidad de Berkeley. A raíz de un articulo publicado en el New York Times titulado "Tomorrow you may be younger”, en 1945, publicó en 1954 su artículo. "Aging: a theory based on free radicals and radiation chemistry" en el Journal of Gerontology, articulo que fue considerado como la expresión de una teoría demasiado simple para explicar el complejo mecanismo del envejecimiento. Actualmente, a los 50 años de su publicación, la teoría de los radicales libres en relación al envejecimiento es universalmente aceptada.

En 1972, Harman y luego en 1980 Miquel, señalaron que en $90 \%$ de los radicales libres se producen a nivel de las mitocondrias, el mismo lugar en que se produce el ATP como fuente de energía. El número de mitocondrias varía según la célula entre 50 y 2.500 , y en todo nuestro cuerpo se acepta que existen unos 10 mil millones de mitocondrias.

Es a nivel de la membrana interna de las mitocondrias donde se produce una reacción o formación de una cadena de transporte de electrones, de tal forma que la molécula de oxígeno $\left(\mathrm{O}_{2}\right)$ pasa a superóxido $\left(\mathrm{O}_{2}-\right)$-radical libre- que a su vez genera el peróxido de hidrógeno $\left(\mathrm{H}_{2} \mathrm{O}_{2}\right)$, y éste al radical hidroxilo ( $\mathrm{OH}-)_{\text {, mientras que la }}$ membrana externa produce solamente $\mathrm{H}_{2} \mathrm{O}_{2}$. El superóxido y el peróxido de hidrógeno proceden de la reducción del oxígeno de la respiración mediante la citocromo-oxidasa mitocondrial, formando parte de un proceso aeróbico fisiológico.

Así pues, los radicales libres son un producto natural del metabolismo aeróbico de la célula, siendo el 2-3\% del oxigeno consumido por la célula el que se convierte en radicales libres. Es a nivel de las mitocondrias donde se metaboliza más del 90\% del oxigeno que consume el cuerpo humano. El 98\% del oxigeno que respiramos lo convierte la mitocondria en ATP y agua, siendo solo el $2 \%$ del oxigeno el que da lugar a la formación de los radicales libres.

Los radicales libres son un subproducto del metabolismo de los alimentos, de la respiración y 
del ejercicio físico, pero asimismo consecuencia de la polución industrial, el consumo de tabaco, de las radiaciones, del uso de pesticidas y aditivos alimentarios Los radicales libres son un mecanismo defensivo, especialmente contra las infecciones bacterianas, pero asimismo son elementos tóxicos, atacando las membranas celulares a nivel de su capa lipídica, alterando su fluidez y elasticidad, con más fácil ruptura de las mismas. Son responsables del depósito de lipofucsina a nivel de la epidermis cutánea (manchas de la piel de los viejos), a nivel de las neuronas y de las células miocárdicas y asimismo actúan contra las proteínas, las enzimas, el tejido conectivo y contra el mismo DNA.

Los radicales libres, en condiciones fisiológicas, son totalmente neutralizados por antioxidantes naturales. Los antioxidantes naturales son tres: la superoxido-dismutasa, la catalasa y la glutation-peroxidasa.

La superóxido-dismutasa(SOD) neutraliza los radicales superóxido $\left(\mathrm{O}_{2}\right.$-), convirtiéndolos en peróxido de hidrogeno $\left(\mathrm{H}_{2} \mathrm{O}_{2} \mathrm{o}\right.$ agua oxigenada), en presencia de zinc:

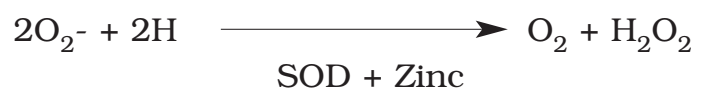

Se ha demostrado una estrecha relación entre los niveles de SOD y el índice de longevidad. La inserción de extra copias del gen SOD a la mosca de la fruta aumenta su expectancia de vida.

Las catalasas reducen el peroxido de hidrogeno a agua y oxigeno molecular:

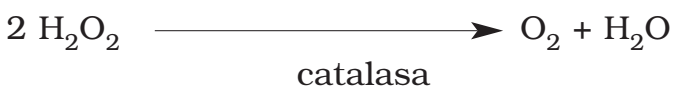

La glutation-peroxidasa (GPH) neutraliza el radical hidroxilo $(\mathrm{OH}-)$, el más tóxico de los radicales libres, a oxígeno en presencia de selenio, actuando como protector de los lípidos de las membranas celulares:

$$
\mathrm{GPH}+\mathrm{OH}^{-} \longrightarrow \mathrm{O}_{2}+\mathrm{H}_{2} \mathrm{O}
$$

La respiración celular se comprende, a modo de resumen, con la descripción de la reacción de Fenton:

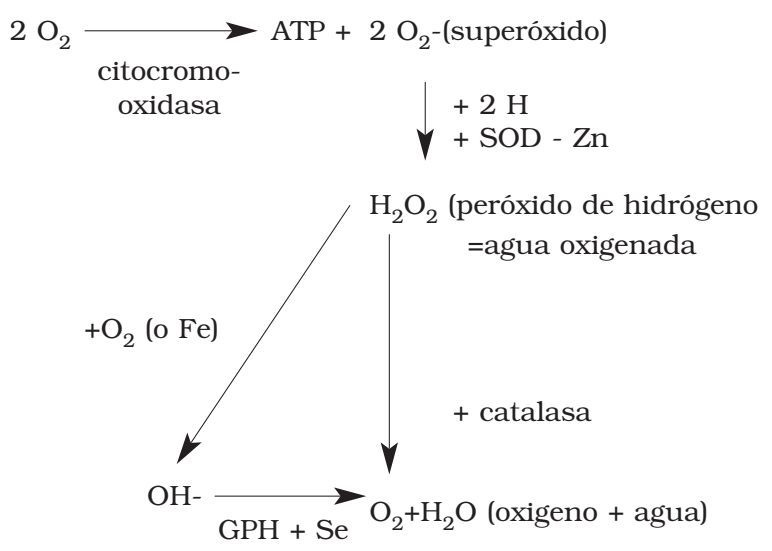

Estas enzimas al actuar como antioxidantes retrasan, o evitan, el proceso de oxidación celular. El antioxidante "al chocar" con el radical libre le cede un electrón, y a su vez se oxida, transformándose en un radical libre más débil, no tóxico, produciéndose una reacción en cascada.

Un radical libre, el superóxido por ejemplo, roba un electrón a un antioxidante, la vitamina $\mathrm{E}$ por ejemplo, y ésta a su vez se transforma en un radical libre mas débil, que roba un electrón a otro antioxidante... Y así sucesivamente hasta neutralizar la acción tóxica del radical libre. Podríamos decir que ocurren como si "el que roba a ladrón, tiene 100 años de perdón”.

La energía producida se almacena en el ATP (adenosin-trifosfato), a nivel de las uniones trifosfato -como una batería cargada- y cuando el organismo precisa gastar energía el ATP pasa a ADP (adenosin-difosfato), semejante a una batería descargada. La mitocondria recarga el ADP y lo pasa nuevamente a ATP. El ejercicio físico da lugar a una mayor producción de ATP y de radicales libres, y es por ello que se aconseja tomar un zumo de naranja después del ejercicio físico, para suministrar una carga suplementaria de antioxidantes.

Se denomina "estrés oxidativo" a la pérdida del equilibrio entre la producción de radicales libres y antioxidantes, considerarlo responsable de:

- envejecimiento: peroxidación de los ácidos grasos de las membranas celulares y daños en el DNA.

- ateroesclerosis: por peroxidación del LDL

- cáncer: por mutaciones del DNA.

- cataratas: por modificación irreversible de proteínas del cristalino. 
- cuadros inflamatorios crónicos: artrosis, artritis reumatoide

Y un gran número de patologías asociadas:

- piel: cáncer, psoriasis

- cerebro: Parkinson, Alzheimer

- digestivo: pancreatitis, úlceras, enfermedad de Crohn

- anemia

- HIV, diabetes

- riñón: IRC

- pulmón: asma, SRAD

- corazón: infarto, trombosis, cardiopatías...

Las mitocondrias, aunque son la principal fuente de producción de radicales libres, no son la única. También los perixosomas (organelas del citosol) generan $\mathrm{H}_{2} \mathrm{O}_{2}$. Asimismo los leucocitos polinucleares cuando se activan por diversas proteínas, como el complemento o las interleuquinas, y en presencia de ión $\mathrm{Fe}$, dan lugar al radical hidroxilo (OH-), lo que particularmente ocurre en los procesos inflamatorios. Por último, la enzima xantina-deshidrogenasa de los endotelios es otra fuente de radicales libres, generalmente de superóxido.

La toxicidad de los radicales libres se debe a que por su acción se altera la función específica de los lípidos, de los aminoácidos y del DNA:

a. La oxidación de los lipidos a nivel de la membrana celular da lugar una alteración de su permeabilidad, con edema y muerte celular. A nivel del LDL -su oxidación- es responsable de la génesis de las placas ateromatosas. Este proceso de peroxidación lipídica da lugar a la producción de un aldehido, el malondialdehido (MDA), que es el que se determina en orina (oxitest) o en plasma. Lo ideal sería averiguar mediante chequeo el estado de oxidación (estado redox) de cada individuo, determinando su nivel de producción de radicales libres y su nivel de producción de antioxidantes, con lo que seríamos capaces de establecer la dieta mas adecuada, modificar sus hábitos de vida y dar los consejos mas oportunos sobre empleo de complementos antioxidantes, es decir cumplir con los requisitos fundamentales de la medicina anti-envejecimiento. Uno de los tests más simples para el estudio del estado redox es el denominado oxitest, en orina y en el que mediante un método colorimétrico se miden los aldehidos resultantes del metabolismo de los radicales libres. Otros métodos mas complejos analizan el estado de oxidación celular a nivel de sangre o plasma.

b. La oxidación de los aminoácidos da lugar a la fragmentación de la proteína a la que ataca, impidiendo su función.

c. La oxidación del DNA produce daño a nivel de los ácidos nucleicos, modificando sus bases, dando lugar a mutaciones a nivel de los oncogenes y/o a nivel de los genes supresores. En la carcinogénesis tiene gran importancia la acción directa de los radicales libres, especialmente del radical hidroxilo sobre el DNA, siendo esta fase considerada como la etapa inicial de la carcinogénesis, seguido de la segunda fase con exposición a los carcinógenos. En el DNA lesionado por mecanismos oxidativos se produce la unión de un grupo metilo a su estructura, siendo este hecho responsable de una mutación genética, y por tanto pudiéndose considerar este hecho como origen del cáncer.

La quimioprevención el cáncer ha alcanzado gran desarrollo, especialmente en relación al cáncer de la próstata, tanto por su alta prevalencia como por su larga latencia y significativa morbilidad y mortalidad. El estudio del efecto de los radicales libres y el uso de antioxidantes es el principal objetivo de la "Chemoprevention Branch of the Division of Cancer Prevention at de National Cancer Institute" de USA. Uno de los estudios de mayor relevancia es el denominado SELECT (Selenium and Vitamin E Chemoprevention Trial) del NCI, iniciado en el año 2002, con resultados que serán valorables en el 2013, cuando se espera poder analizar los resultados de 32.400 hombres de 55 años de edad, con tacto rectal y PSA normales, en relación a la aparición de cáncer de próstata con dietas suplementadas con alfatocoferol, selenio, ambos productos, o placebo por un periodo mínimo de siete años y máximo de doce.

La medicina anti-envejecimiento no ha logrado crear un "elixir de juventud", pero si establecer dietas, como la dieta mediterránea, hábitos de vida y complementos antioxidantes, que por un lado lleven a la producción de la menor cantidad posible de radicales libres y por otra parte logren aporte suplementario de antioxidantes. 
La dieta mediterránea, rica en frutas y verduras, aceite de oliva virgen, nueces, tomates y vino, constituye un rica fuente de antioxidantes. Las nueces contienen ácido alfa-linoleico, ácido graso omega tres, 1-arginina y gamma-tocoferol. Las nueces contribuyen a restaurar la función endotelial, tienen efectos cardioprotectores, reducen el colesterol total y especialmente el LDL, restituyen la elasticidad a las arterias rígidas y aumentan su flujo Se puede decir que las nueces tienen efecto similar a un medicamento, formando parte de los productos llamados "nutricéuticos".

Como oxidantes tienen asimismo un importante papel la vitamina E, el selenio, el licopeno, la vitamina $\mathrm{C}$, el betacaroteno, la soja, la ubiquinona o vitamina $\mathrm{Q}$, etc.

La vitamina E natural o alfa-tocoferol es liposoluble, y actúa protegiendo los lípidos de las membranas de las neuronas, de los músculos y de los vasos sanguíneos. Se encuentra en el maíz, las nueces, las aceitunas, los vegetales de hoja verde, los aceites vegetales y el germen de trigo. $\mathrm{El}$ selenio facilita la función de la vitamina $\mathrm{E}$, por lo que deben asociarse. El selenio protege a las células frente al radical superóxido. Se considera que su déficit se asocia a un mayor riesgo de cáncer de próstata, el cual, a su vez, disminuye los niveles sanguíneos de selenio, por lo que se debe administrar no sólo como elemento preventivo de dicho cáncer sino asimismo en los pacientes con cáncer de próstata, dado que estos pacientes, incluso con dieta adecuada, pueden presentar bajos niveles de selenio.

El licopeno, importante antioxidante de la familia de los carotenoides, existe en gran cantidad en los tomates y se considera que tiene un efecto protector frente al cáncer de la próstata. $\mathrm{El}$ betacaroteno -retinol- precursor de la vitamina A, protege a los neutrófilos frente los radicales libres producidos en la reacción inflamatoria, sin alterar su capacidad destructora bacteriana.

La vitamina $\mathrm{C}$, hidrosoluble, actúa como un potente antioxidante frente los lipidos proteicos y regenera los radicales oxidados de la vitamina $\mathrm{E}$, cediéndole un electrón, para devolverla a la forma antioxidante.

Tal como anteriormente hemos descrito el proceso de oxidación y su neutralización por los antioxidantes se produce mediante una reacción en cadena con robo o trasvase de electrones de un antioxidante al otro. $\mathrm{Y}$ así la glutatio-peroxidasa, al actuar sobre el radical hidroxilo, se oxida, y se regenera gracias a la glucosa. La vitamina $\mathrm{E}$ al neutralizar dicho radical hidroxilo se transforma en un antioxidante menos potente, regenerándose gracias a la vitamina C, que su vez es regenerada por el ácido lipoico, y éste por la glucosa.

Frente los indudables beneficios de los antioxidantes cabe también considerar que un exceso de los mismos puede ser perjudicial. En efecto, a niveles altos, el selenio, la vitamina A y el ión hierro son tóxicos, por lo que únicamente se deberían administrar antioxidantes cuando se demostrara su déficit mediante el estudio del perfil redox y el perfil antioxidante en forma individual.

El problema de la posible toxicidad disminuye cuando estos antioxidantes se administran a personas de edad avanzada, en los que, con toda seguridad, existe un cierto grado de "mala nutrición", con déficit proteico, déficit calórico, déficit de minerales y vitaminas, junto a una posible alteración hidroelectrolítica.

Al ser preguntado el doctor Harman -el descubridor de los radicales libres- sobre que suplementos toma personalmente, indicó: $200 \mathrm{mg}$ diarios de vitamina $\mathrm{E}, 10 \mathrm{mg}$ en cada comida de coenzima $\mathrm{Q}$ (ubiquinona), dos veces al día $20 \mathrm{mg}$ de selenio y una tableta diaria de un multivitamínico.

Otro aspecto interesante en cuanto a las causas del envejecimiento es su relación con la restricción calórica y la denominada "glicación proteica"

La unión de moléculas de glucosa o fructosa a las proteínas da lugar al proceso llamado "glicación" o "caramelización proteica". En inglés se conoce por la sigla AGEs ("Advanced Glication Endproducts"). Las proteínas glicadas son patógenas:

- si son enzimas, pierden su actividad

- si son proteínas estructurales, dan lugar a alteraciones tisulares

- si son inmunoglobulinas, no actúan como anticuerpos (patologías por autoinmunidad).

La producción de AGEs es un mecanismo fisiológico habitual y el organismo tiene medios de defensa. Lo realmente peligroso es la presen- 
cia de picos de glucemia elevada, responsables de las típicas lesiones de la nefropatía y retinopatía diabética. La diabetes tipo II, que sufre un $25 \%$ de la población adulta, y debida a un aumento de la secreción de insulina con hiperglicemia (diabetes resistente a insulina), es la principal responsable de la sobreproducción de AGEs, lo que contribuye a acelerar el envejecimiento y a la mayor frecuencia de enfermedades degenerativas como el Alzheimer.

La restricción calórica modifica la resistencia a la insulina. La disminución de la ingesta de calorías es otro de los mecanismos para retrasar o enlentecer el proceso de envejecimiento. El llamado plan CRAN ("Caloric Restriction with Adequate Nutrition") consiste en suministrar una dieta baja en calorías -esencialmente vegetariana- que seguida teóricamente durante más de dos décadas se considera que lograría aumentar 3,6 años la expectancia de vida.

El CRAN se ha demostrado que alarga la expectancia de vida en gusanos, moscas, roedores y primates, consiguiendo en algunos casos con una reducción calórica de un 40\% aumentar en un $65 \%$ la supervivencia, siendo una incógnita lo que pueda ocurrir con los seres humanos. La disminución de la ingesta de calorías da lugar a una disminución de la temperatura corporal, una disminución de la insulina plasmática y un aumento de los niveles de DHEAS.

El por qué el CRAN aumenta la expectancia de vida se explica por el hecho de que el bajo nivel de utilización calórica da lugar a una disminución del gasto energético, con menos producción de radicales libres y niveles más bajos de glucemia. El objetivo teórico es encontrar que mediante manipulación genética o gracias a una terapéutica medicamentosa se consiguieran los efectos del CRAN "sin pasar hambre".

La manipulación genética se demostró efectiva en el aumento de la longevidad del gusano "Caenorhabditis elegans". Aumenta su vida de 16 a 48 días al disminuir la expresión del gen DAF-2, y también con la adición del gen SIR-2. En cuanto a la manipulación farmacológica son numerosos los artículos científicos que atribuyen a la Metformina, medicamento usado en el tratamiento de la diabetes tipo 2 , efectos semejantes a la restricción calórica, siendo aún una incógnita el posible aumento de la expectancia de vida.
Lo que es evidente es que la obesidad disminuye la expectancia de vida, de tal forma que un índice de masa corporal superior a 40 triplica la mortalidad, si bien en los mayores de 70 años, un aumento moderado de masa corporal (alrededor de un $30 \%$ ), no es un factor de riesgo. Un ejemplo de la efectividad de la restricción calórica en relación a la supervivencia lo ofrecen las estadísticas de la población de Okinawa, que con una dieta con un $20 \%$ menos calorías que el resto de japoneses, presentan un $41 \%$ menos accidentes vasculares, con un $31 \%$ de reducción en el número de cánceres y un mayor número de centenarios.

La medicina anti-envejecimiento tiene como principales objetivos:

- evitar la formación excesiva de radicales libres

- aportar antioxidantes, para una mejor neutralización de los radicales libres

- evitar picos de hiperglicemia en la producción de AGEs

- corregir los excesos de colesterol LDL

-y principalmente, evitar la obesidad

El urólogo debe hacer recomendaciones a los que sufren el denominado sindrome metabólico o sindrome X, caracterizado por presentar:

- resistencia a la insulina (diabetes tipo 2)

- triada lipídica anormal:

- aumento de trigliceridos

- aumento del colesterol LDL

- disminución del colesterol HDL

- hipertensión arterial

- estado precoagulación

- OBESIDAD.

Y, para terminar con esta presentación, algunas reflexiones:

- "todo depende de ti: el envejecimiento es una responsabilidad personal, que debe asumirse a partir de los 45 años".

- Existe el peligro de que al conseguir alargar la vida creemos Thitonus. En la mitología griega se describe que la diosa Aurora -inmortalsolicita de su padre Zeus, que haga inmortal a su esposo. Zeus accede, le concede la inmortalidad, pero sin concederle la "eterna juventud", por lo que al envejecer, su esposa -la diosa- lo convierte en saltamontes, hoy en día símbolo de la inmortalidad en la cultura china . 
- En el futuro la enfermedad desaparecerá. La muerte será el fin fisiológico del envejecimiento. En último período de la vida será un período de buena salud.

- Nunca se es demasiado viejo para ser más joven. Vivir es la gran fiesta de la vida, si se saben aceptar las limitaciones que la edad impone (capacidad de adaptación).

- "Vivir plenamente", hasta que llegue el momento. Hay que aprender a envejecer.

- Agustí Altisent monje del monasterio de Poblet, publicó en 1994: "las rodillas se me empiezan a doblar, pierdo la memoria, tengo menos facultades...Estoy muriendo a plazos. Afortunados los que mueren al contado".

- Y, como hemos visto al principio, recordemos que envejecer empieza al nacer, y que lo que es seguro es que ahora al terminar la lectura de esta comunicación todos seremos más viejos que cuando la empezamos.

\section{BIBLIOGRAFIA RECOMENDADA}

Pray LA. Epigenetics: genome, meet your environment. The Scientist. 14-20. july 2004.

Harman D. Aging:a theory based on free radicals and radiation chemistry. J.Gerontol 1956;11:298-300.

Harman D. The biologic clock: the mitochondria?. J Am Geroltol Soc 1972;20:145-147.

Sastre J. Pallardó FV,Viñas J. The role of mitochondrial oxidative stress in aging. Free Radical Biology \& Medicine 2003;35:1-8.

Lin MT, Beal MF. The oxidative damage theory of aging.. Clinical Neuroscience Research 2003;2:305-315.
Mechanisms of aging:the importance of AGEs. http://www.benbest.com/lifeext/aging.html.

González Sansejo ML, Muñiz P. Actividad antioxidante de los alimentos. Vall Bellés.Iberica 2004;85/472:151-157.

Proceso de envejecimiento,¿Cómo frenarlo?. Nota informativa 48. http: / / www.sabater-tobella.com/index.php?.id=228\&L=0.

Thrichopoulou A. et al. Modified Mediterranean diet and survival: EPIC-eldely prospective cohort study. BMJ 2005; 330:991.

Romero Cagigal I. Polifenoles del vino tinto inhiben la proliferación e inducen apoptosis en células de cáncer de próstata LNCaP. Tesis Doctoral 2004.

Marrugat J, Covas MI, Fito M, Schroder H, Miro-Casas E, Gimeno E. Effects of differing phenolic content in dietary olive oil on lipids and LDL oxidation:a randomized clinical trial. European Journal of Nutrition 2004;43:140-147.

The metabolic syndrome.The roots of aging. An interview with Dr. D. Harman. Life extension Magazine. http://www.lef.org.

Articles about caloric restriction with adequate nutrition (CRAN). http://www.benbest.com/calories/clories.html.

Klein EA,Thompson IM. Update on chemoprevention of prostate cancer. Current Opinion in Urology 2004;14(3):143-149.

Klein EA, Lippman SM, Thompson IM, Goodman PJ, Albanes $\mathrm{D}$, Taylor PR, et al. The Selenium and Vitamin E Prevention Trial World J-Urol 2003;21:21-23.

Dr. F.J. Solé Balcells

Servicio de Urología

Fundació Puigvert

Cartagena, 340-350

08025 Barcelona

E-mail: sole@pulso.com

(Trabajo recibido el 16 de enero 2006) 